\title{
LOOSELY BERNOULLI CARTESIAN PRODUCTS
}

\author{
LAIF SWANSON ${ }^{1}$
}

\begin{abstract}
For any totally ergodic loosely Bernoulli automorphism $T$, a class $\delta(T)$ of loosely Bernoulli automorphisms is constructed. Each class $\delta(T)$ includes zero entropy automorphisms which do not have discrete spectrum, and has the property that for $S \in \delta(T)$, the Cartesian product $T \times S$ is loosely Bernoulli.
\end{abstract}

1. A lot of attention in ergodic theory has been paid to loosely Bernoulli automorphisms since Jack Feldman defined them in 1975. He showed that being loosely Bernoulli was a necessary condition for an automorphism to be Kakutani equivalent to a Bernoulli shift or circle rotation. Ben Weiss and Don Ornstein showed sufficiency in their development of the "equivalence theory". They also showed that for different positive entropies, inducing is the same as Kakutani equivalence; that finite rank automorphisms are loosely Bernoulli; and that the Cartesian product of an automorphism with discrete spectrum with a zero entropy loosely Bernoulli automorphism is loosely Bernoulli if ergodic. This last yields the fact that powers of zero entropy loosely Bernoulli automorphisms are loosely Bernoulli if ergodic; Dan Rudolph showed this for automorphisms with positive entropy [6]. Don Ornstein gave an example of a loosely Bernoulli automorphism with zero entropy whose Cartesian produce with itself is not loosely Bernoulli [5].

Except for automorphisms with discrete spectrum and Bernoulli shifts, nothing general is known about when the Cartesian product of two automorphisms is loosely Bernoulli. What class $\mathcal{T}_{1}$ of automorphisms has the property that $S \in \mathscr{T}_{1}$ and $T$ loosely Bernou'li implies that $S \times T$ is loosely Bernoulli? Is there a largest class $\mathscr{T}_{2}$ with the property that $S$ and $T \in \mathscr{T}_{2}$ implies that $S \times T$ is loosely Bernoulli? (It is not even immediately clear that there are any weakly mixing automorphisms $S$ and $T$, neither a Bernoulli shift, such that $S \times T$ is loosely Bernoulli.) What is the class $\mathscr{T}_{3}$ such that $T \in \mathscr{T}_{3}$ implies $T \times T$ loosely Bernoulli; in particular, does it contain automorphisms besides Bernoulli shifts?

Several years before loosely Bernoulli was defined, Katok and Stepin categorized classes of zero entropy automorphisms by periodic approximation. We show that each class includes weakly mixing automorphisms (though

Received by the editors November 28, 1977 and, in revised form, April 3, 1978.

AMS (MOS) subject classifications (1970). Primary 28A65, 60G10.

Key words and phrases. Measure preserving transformations, induced automorphisms, loosely Bernoulli, entropy.

'Partially supported by NSF grant MCS 76-06005. 
the intersection of the classes is contained within the discrete spectrum automorphisms), and that to each totally ergodic loosely Bernoulli automorphism $T$ is associated one of these classes $\delta(T)$ with the property that if $S \in \mathcal{S}(T), S \times T$ is loosely Bernoulli.

This eliminates the possibility for a "universally bad" loosely Bernoulli automorphism: a $T$ with the property that if $S \times T$ is loosely Bernoulli, then $S$ must have discrete spectrum or be a Bernoulli shift.

In order to identify the class $\mathcal{T}_{1}$ of automorphisms $S$ with the property that $S \times T$ is loosely Bernoulli whenever $T$ is, one would want the opposite construction: for each $T$ not in $\mathcal{T}_{1}$, a family $\mathcal{S}^{\prime}(T)$ of loosely Bernoulli automorphisms with the property that $S^{\prime} \times T$ is not loosely Bernoulli for $S^{\prime} \in \mathcal{S}^{\prime}(T)$. While it seems likely that the automorphisms with discrete spectrum and the Bernoulli shifts may be all the automorphisms in this class, such a class of examples does not now exist. Another similar question which might be of interest is when the Cartesian products of non-Bernoulli $K$-automorphisms are loosely Bernoulli.

\section{Loosely Bernoulli automorphisms.}

Definition (Feldman). If $\omega, \omega^{\prime} \in\{1,2, \ldots, m\}^{n}$, then $\bar{f}_{n}\left(\omega, \omega^{\prime}\right)=1-$ $k / n$,where $k$ is the maximal integer for which we can find sequences $i_{1}<i_{2}$ $<\cdots<i_{k}, j_{1}<j_{2}<\cdots<j_{k}$ with $\omega\left(i_{r}\right)=\omega^{\prime}\left(i_{r}\right), 1 \leqslant r \leqslant k$. If $\mu_{n}, \mu_{n}^{\prime}$ are two probability measures on $A^{n}=\{1,2, \ldots, m\}^{n}$, then $\bar{f}_{n}\left(\mu_{n}, \mu_{n}^{\prime}\right)=\inf \{\varepsilon$ : there is a measure $\lambda$ on $A^{n} \times A^{n}$ whose marginals are $\mu_{n}, \mu_{n}^{\prime}$, and for $\varepsilon$-a.e. element $\left(\omega, \omega^{\prime}\right)$ of $A^{n} \times A^{n}$ with respect to $\lambda$, we have $\bar{f}_{n}\left(\omega, \omega^{\prime}\right)<\varepsilon$.

Definition. A process $(T, P)$ is loosely Bernoulli if for any $\varepsilon>0$ there exists an $N$ such that for any $n \geqslant N$ and any $m, \bar{f}\left(\left\{T^{i} P\right\}_{i}^{n},\left\{T^{i} P / A\right\}_{1}^{n}\right)<\varepsilon$ for E-a.e. $A \in \bigvee_{-m}^{0} T^{i} P$.

The definition above is equivalent to the existence of an $n$ such that for any $m, \bar{f}_{n}\left(\left\{T^{i} P\right\}_{1}^{n},\left\{T^{i} P / A\right\}_{1}^{n}\right)<\varepsilon$ for $\varepsilon$-a.e. $A \in \bigvee_{-m}^{0} T^{i} P$.

THEOREM [see [7]]. If $(T, P)$ is loosely Bernoulli for some generator $P$, then $(T, Q)$ is loosely Bernoulli for all finite partitions $Q$.

In case $(T, P)$ is loosely Bernoulli for some generator $P, T$ is called loosely Bernoulli.

Definition. If $A$ is a positive measure subset of $X, T$ an automorphism of $X$, let $n(x)=\min \left\{m>0: T^{m} x \in A\right\}$, where $x \in A$. The induced automorphism $T_{A}$ is defined by $T_{A}(x)=T^{n}(x)$ [2].

Definition. Automorphisms $S$ and $T$ are Kakutani equivalent if they induce a common automorphism.

If $S$ and $T$ are Kakutani equivalent, then both are of the same entropy class, i.e. both have zero entropy, both have positive finite entropy, or both have infinite entropy. Feldman showed, by constructing a positive entropy automorphism which is not loosely Bernoulli and showing that only loosely Bernoulli automorphisms can be Kakutani equivalent to Bernoulli shifts, that 
Kakutani equivalence classes are finer than entropy classes.

Ornstein and Weiss showed that if $0<h(T)<h(S)<\infty$ and if $S$ and $T$ are Kakutani equivalent, then $T$ induces $S$, and that a loosely Bernoulli automorphism of positive, finite entropy is Kakutani equivalent to a Bernoulli shift.

3. Approximation by periodic transformations. Katok and Stepin consider approximation by periodic transformations as an invariant of automorphisms. We will use such automorphisms in our construction.

Definition. An automorphism $T$ is said to admit periodic approximation with speed $f$ ( $f$ a sequence) if there is a sequence of finite, measurable partitions $\xi_{m}=\left(C_{m}^{1}, C_{m}^{2}, \ldots, C_{m}^{q(m)}=C_{m}^{0}, D_{m}\right)$ with the properties

(1) $\xi_{m} \rightarrow \varepsilon$, the partition into points, as $m \rightarrow \infty$,

(2) $\sum_{i=0}^{q(m)-1} \mu\left(T C_{m}^{i} \triangle C_{m}^{i+1}\right) \leqslant f(q(m))$.

If $T$ admits periodic approximation with speed $f(n)$, where $f(n)=o(g(n))$, then $T$ is said to have periodic approximation with speed $o(g(n))$.

It is clear that automorphisms which admit periodic approximation with speed $o(1 / n)$ are rank one. This implies that they are zero entropy loosely Bernoulli automorphisms [7]. (Katok uses "good periodic approximation" to describe this property of periodic approximation with speed $o(1 / n)$. He is able to characterize these automorphisms by use of a metric between $\bar{f}$ and $\bar{d}$.) We are interested only in those approximation orders which are at least that good, and so we will assume throughout that $f(n)=o(1 / n)$. This means that we can think of most names for the automorphisms we are studying as looking like finite rotations most of the time.

What does that mean about the ergodic properties of these automorphisms? Katok and Stepin have shown that if $T$ admits approximation by periodic transformations with speed $f$ for every sequence $f$ then $T$ has discrete spectrum and its eigenvalues are all roots of unity, and that if $T$ has good periodic approximation then $T$ has no mixing factors. Thus every weakly mixing automorphism fails to admit a periodic approximation with speed $f$ for some sequences $f$, and automorphisms with good periodic approximation can never be mixing. The theorem below shows that, on the other hand, for every $f$ there are weakly mixing automorphisms which admit periodic approximation with speed $f$.

THEOREM. Given a sequence $f$, there are weakly mixing automorphisms which admit periodic approximation with speed $f$.

Proof. Recall that we are assuming (WLOG in this case) that $f(n) \rightarrow 0$. We will construct an automorphism $T$ which admits periodic approximation of order $f$ and then show that it is weakly mixing.

The automorphism is constructed by cutting and stacking of gadgets. We start with a single interval, $G(1)$, so the height $h(1)=1$. If we have $G(n)$, we get $G(n+1)$ by dividing $G(n)$ into $k=2([1 / f(n)]+1)$ thin stacks, stacking 
them one on top of another, and adding one interval to the top. Since $k$ is at least two, we have defined an automorphism $T$ of a finite measure space, which we can of course normalize to a probability space with probability measure $\mu$. Notice that $h(n+1)=k h(n)+1$.

It is clear from the construction that $T$ admits periodic approximation with speed $f$, with $\xi_{n}$ the partition into the intervals of $G(n)$. Since $T$ has rank one, it is ergodic. To see that $T$ is weakly mixing, assume not; let $g$ be an eigenfunction, $|g|=1$, with eigenvalue $\lambda$. We will see, by a standard argument, that $\lambda=1$. Since the $G(n)$ 's generate, given a small $\varepsilon>0$ there is an $n$, an interval $C_{n}^{l}$ of $G(n)$, and a $\gamma$ with the property that $|g(x)-\gamma|<\varepsilon / 6$ for all $x \in C_{n}^{l}$ except a set of $x$ 's of measure at most $\varepsilon \mu\left(C_{n}^{l}\right)$. Because of the action of $T$ on $G(n)$, this gives a value $\gamma_{1}=\lambda^{-l+1} \gamma$ and a set $M \subset C_{n}^{1}, \mu(M)>(1-$ $\varepsilon) \mu C_{n}^{1}$, with $\left|g(x)-\gamma_{1}\right|<\varepsilon / 6$ for $x \in M$. That means, looking in $G(n+1)$, that, for $x \in M \cap T^{-i h(n)} M,\left|g\left(T^{i h(n)} x\right)-\gamma_{1}\right|<\varepsilon / 6$, and $\left|g(x)-\gamma_{1}\right|<\varepsilon / 6$, so

$$
\begin{aligned}
\left|\lambda^{i h(n)}-1\right| & =\left|\lambda^{i h(n)} g(x)-g(x)\right|=\left|g\left(T^{i h(n)} x\right)-g(x)\right| \\
& \leqslant\left|g\left(T^{i h(n)}\right) x-\gamma_{1}\right|+\left|g(x)-\gamma_{1}\right|<\varepsilon / 6+\varepsilon / 6=\varepsilon / 3 .
\end{aligned}
$$

But for most $i, 1 \leqslant i \leqslant k$, there is some $x \in M \cap T^{-i h(n)} M$. Thus there is some $i$ and $j$ with $i+j=k$ for which $\left|\lambda^{i h(n)}-1\right|<\varepsilon / 3$ and $\left|\lambda^{j h(n)}-1\right|<$ $\varepsilon / 3$, and so $\left|\lambda^{k h(n)}-1\right|<2 \varepsilon / 3$.

But now looking in $G(n+2)$, we see that $M \cap T^{-h(n+1)} M$ is not empty, and for $x \in M \cap T^{-h(n+1)} M,\left|\lambda^{h(n+1)} g(x)-g(x)\right|<\varepsilon / 3$ and so $\mid \lambda^{h(n+1)}-$ $1 \mid<\varepsilon / 3$. But $h(n+1)=k h(n)+1$, and so

$$
\begin{aligned}
|\lambda-1| & =\left|\lambda^{h(n+1)}-\lambda^{k h(n)}\right| \leqslant\left|\lambda^{h(n+1)}-1\right|+\left|\lambda^{k h(n)}-1\right| \\
& <\varepsilon / 3+2 \varepsilon / 3=\varepsilon .
\end{aligned}
$$

4. Cartesian products. Little is known about when Cartesian products are loosely Bernoulli. We will see that every loosely Bernoulli automorphism is a direct factor (with a weakly mixing non-Bernoulli automorphism) in a loosely Bernoulli automorphism.

First, even though its existence follows from the later theorem, it is worthwhile for seeing what is going on to construct an example. Let $S$ be an automorphism, constructed by cutting and stacking with stack heights $h(n)$, admitting periodic approximation of order $o\left(1 / n^{2}\right)$. Let $T$ be such an automorphism as well, with each of its stack heights $h^{\prime}(n)$ about the same as the corresponding one for $S$ but relatively prime to it. Then most $h(n) h^{\prime}(n) S \times T$ names will cycle through all the names in the product of the $n$th gadgets (since the product of relatively prime rotations is a rotation), and the error in the approximation is $o$ of the product height. Thus a zero entropy product automorphism $S \times T$ can be loosely Bernoulli, even with both $S$ and $T$ weakly mixing.

In fact, given any totally ergodic loosely Bernoulli $S$ there is a class $\mathcal{S}(S)$ 
of automorphisms, some weakly mixing with zero entropy and some weakly mixing with positive entropy, with the property that $S \times T$ is loosely Bernoulli for all $T \in \delta(S)$. We construct that $\delta(S)$ by constructing a sequence $f$ (depending on $S$ ) with the property that if $T$ has periodic approximation with speed $f$, then $S \times T$ is loosely Bernoulli. The key to the construction is the result (from Ben Weiss' notes in case $h(S)=0$, and by Dan Rudolph [6] in case $h(S)>0$ ) that ergodic products of loosely Bernoulli automorphisms with finite rotations are loosely Bernoulli, together with the fact that automorphisms admitting periodic approximation are very much like finite rotations.

THEOREM. If $S$ is a totally ergodic loosely Bernoulli automorphism, then there is an $f$ with the property that if $T$ admits periodic approximation of order $f$, then $S \times T$ is loosely Bernoulli.

Proof. Fix a partition $P$ generating $S$. Denote by $R_{n}$ the rotation on $n$ letters and let $Q_{n}$ be the partition into these $n$ letters. Since $S$ is totally ergodic, $S \times R_{n}$ is ergodic and so loosely Bernoulli. That means that for every $n \in \mathbf{N}, \varepsilon>0$, there is an $M(n, \varepsilon)$ with the property that if $m \geqslant M(n, \varepsilon)$, $k>0$, and $A, B \varepsilon / 2$-almost any atoms in $\bigvee_{j=-k}^{0}\left(S \times R_{n}\right)^{j}\left(P \times Q_{n}\right)$, then

$$
\bar{f}\left(\left\{\left(S \times R_{n}\right)^{i}\left(P \times Q_{n}\right) / A\right\}_{1}^{m},\left\{\left(S \times R_{n}\right)^{i}\left(P \times Q_{n}\right) / B\right\}_{1}^{m}\right)<\varepsilon .
$$

Let $\left\{\varepsilon_{n}\right\}_{n}$ be any sequence with $\varepsilon_{n} \rightarrow 0$, and define $f(n)=\varepsilon_{n} / M\left(n, \varepsilon_{n}\right)$.

We will show that if $T$ admits periodic approximation of order $f$ (and so has an associated sequence $q(n)$ of stack heights) that $S \times T$ is loosely Bernoulli; that is, that futures of the sets of strings with two different fixed pasts can be $\bar{f}$ matched.

Given $\varepsilon$, choose $n$ such that $\varepsilon_{q(n)}<\varepsilon / 2$. Look at two past names for $S \times T$ strings. Each determines a past for $S$ together with a particular $T$ name. Now look at the futures to $M\left(q(n), \varepsilon_{q(n)}\right)$. Because $T$ has periodic approximation of order $\varepsilon_{n} / M\left(n, \varepsilon_{n}\right)$, for all but $\varepsilon_{q(n)}<\varepsilon / 2$ of the $T$ names, this looks like a rotation on $q(n)$ elements. That means that most past names for $S \times T$ determine a future name just like past names for $S \times R_{q(n)}$ do. But by our choice of $M(n, \varepsilon)$, these names can be matched. Q.E.D.

If $T$ with positive entropy is desired, just cross one of these automorphisms with a Bernoulli shift.

In case $S$ is not totally ergodic, a similar construction can be made if $S^{p}$ fails to be ergodic only when $p$ has common divisors with some fixed $j$. Then one must pick $T$ with stack heights relatively prime to $j$, but that is easy.

5. The remaining questions. Several of the questions at the beginning remain. Is there a zero entropy $T$ (or any non-Bernoulli $T$ ) with $T \times T$ loosely Bernoulli? For loosely Bernoulli automorphisms $S$ does there exist a loosely Bernoulli automorphism $T$ (depending on $S$, of course) with $S \times T$ not loosely Bernoulli? Which of these results (positive or negative) work for 
other ergodic properties? (For example, for each loosely Bernoulli $S$ we have constructed a family of automorphisms $T$ such that $S \times T$ is loosely Bernoulli. But $T$ is not mixing. Could it be? Could it be $K$ ?) Can any of this help in finding out about loose Bernoullicity of "natural" automorphisms?

ADDED IN PROOF. The first question has been answered by A. B. Katok.

\section{BIBLIOGRAPHY}

1. J. Feldman, New K-automorphisms and a problem of Kakutani, Israel J. Math. 24 (1976), 16-37.

2. S. Kakutani, Induced measure preserving transformations, Proc. Imp. Acad. Tokyo 19 (1943), 635-641.

3. A. B. Katok, Change of time, monotone equivalence and standard dynamical systems, Dokl. Akad. Nauk SSSR 223 (1975), 789-792.

4. A. B. Katok and A. M. Stepin, Approximations in ergodic theory, Russian Math. Surveys 22 (1967), 77-102.

5. D. Rudolph, Nonequivalence of measure preserving transformations, Lecture Notes, 1976.

6. __ If a two-point extension of a Bernoulli shift has an ergodic square, then it is Bernoulli (to appear).

7. B. Weiss, Equivalence of measure preserving transformations, Lecture Notes, The Institute for Advanced Studies of the Hebrew University of Jerusalem, 1976.

Department of Mathematics, Texas A\&M University, College Station, Texas 77843 